\title{
USO DE MODELOS FÍSICOS EN EL APRENDIZAJE Y APLICACIÓN DE LA FÍSICA
}

\author{
Moisés Segundo Sánchez Arteaga ${ }^{1}$
}

\section{RESUMEN}

En el presente trabajo se muestra una forma sencilla para aplicar las leyes de la física en el análisis de un fenómeno, no se requiere que el estudiante o investigador invierta tiempo en cálculos matemáticos como la derivación, integración e incluso resolución de ecuaciones diferenciales, lo cual le podría con llevar a distraerse del problema físico principal e incluso a veces hasta no encontrar soluciones analíticas a sus modelos.

Se presenta una serie de casos típicos de la física general, que con un software simple como el modellus y muy poquísimas ecuaciones, se puede obtener respuesta a los modelos planteados y hacer el análisis correspondiente en términos de los parámetros requeridos.

Palabras claves: Modelo físico, simulación, leyes físicas, resolución de ecuaciones.

\section{SUMMARY}

In this paper a simple way to apply the laws of physics in the analysis of a phenomenon is shown, it is not required that the student or researcher spend time on mathematical calculations such as derivation, integration and even solving differential equations, which you may be distracted to carry the main physical problem and sometimes not find analytical solutions to their models.

1 Área Académica de Física. Facultad de Ingeniería. Universidad Ricardo Palma. moises.sancheza@urp.pe 
Several of typical cases of general physics, are presented with a simple software like Modellus and very few equations, you can get answers to the proposed models and make the corresponding analysis in terms of the required parameters is presented.

Keywords: Physical model, simulation, physical laws, equation solving.

\section{INTRODUCCIÓN}

Cuando estudiamos cierto fenómeno físico, afín de conocer las leyes que participan, los parámetros que intervienen, la influencia de estos en el fenómeno y hacer pronósticos para casos concretos, debemos seguir un proceso natural científico con la siguiente secuencia: Identificar el fenómeno a estudiar, indagar sobre las leyes físicas participantes, tener información de las cantidades físicas que participan tratando de cuantificarlas y formular un modelo físico que integre esta información para luego darle solución a las ecuaciones resultantes y el análisis correspondiente.

Por lo general la parte de este proceso que consume más tiempo y que muchas veces no hay las herramientas suficientes para superarlo se refiere a la resolución de las ecuaciones que resulten de la aplicación del modelo físico. Se pueden presentar integrales que no tienen solución analítica o si la tienen podría ser tediosa su solución con expresiones complejas; también pueden presentarse ecuaciones diferenciales lineales o no lineales cuya solución analítica puede ser compleja y adicionalmente algunas veces se tendrá que recurrir a una sucesión de aproximaciones matemáticas.

Todo esto conlleva un esfuerzo adicional al estudiante o investigador en el desarrollo del modelo físico y algunas veces no consigue tener una solución matemática analítica como espera.

Avanzando de la mano con la tecnología, algunos métodos de calculo que solían hacerse a mano son ahora obsoletos, como por ejemplo obtener raíces cuadradas y cubicas, solución de ecuaciones algebraicas, 
trabajo con matrices y en los últimos tiempos derivaciones, integración y resolución de ecuaciones diferenciales; actualmente las calculadoras en sus diferentes modalidades ayudan a resolver estas situaciones.

La tecnología actual nos permite simplificar el desarrollo de los modelos físicos dándonos mayor rapidez y precisión en los resultados numéricos de nuestros fenómenos a estudiar.

Presentamos varios casos típicos en la física general que en vez de resolverlos analíticamente con procedimientos matemáticos convencionales, los resolvemos con el uso del software modellus que como muchos otros (Pej. Math Lab. Stella, Math Cad, etc.), podemos utilizar muy pocas ecuaciones propias de las leyes físicas, que nos brindan una solución y análisis de la evolución del fenómeno así como la influencia de los diferentes parámetros participantes.

\section{MATERIAL Y MÉTODOS}

Se presentan varios casos típicos en la enseñanza de la física en el cual se muestra una solución sencilla utilizando el software Modellus versión 4.0. (Vitor Duarte Teodoro, 1996).

1. Fuerza dependiente de la posición: Ley de coulomb, gravitatoria. $\mathbf{F}=\mathbf{K} / \mathbf{r}^{2}$. (Sears - Zemansky - Young - Freedman. 2005).

Una carga puntual positiva $\mathrm{q}$ se lanza con una velocidad inicial $\mathrm{V}_{0}$ hacia un núcleo de carga $Q$. Se estudia el movimiento de la carga puntual sujeta a la fuerza eléctrica de repulsión la cual depende de la distancia entre ambas.

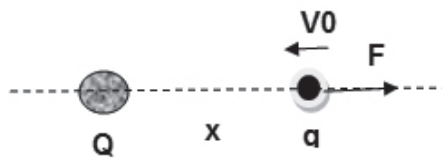

Fuerza eléctrica: $\vec{F}=\frac{k Q q}{r^{2}} \hat{u}_{r}$

Segunda Ley de Newton: $-\frac{k Q q}{x^{2}}=m \frac{d^{2} x}{d t^{2}}$

Ecuación diferencial por resolver: $\frac{d^{2} x}{d t^{2}}+\frac{c}{x^{2}}=0$; siendo $c=\frac{k Q q}{m}$ 

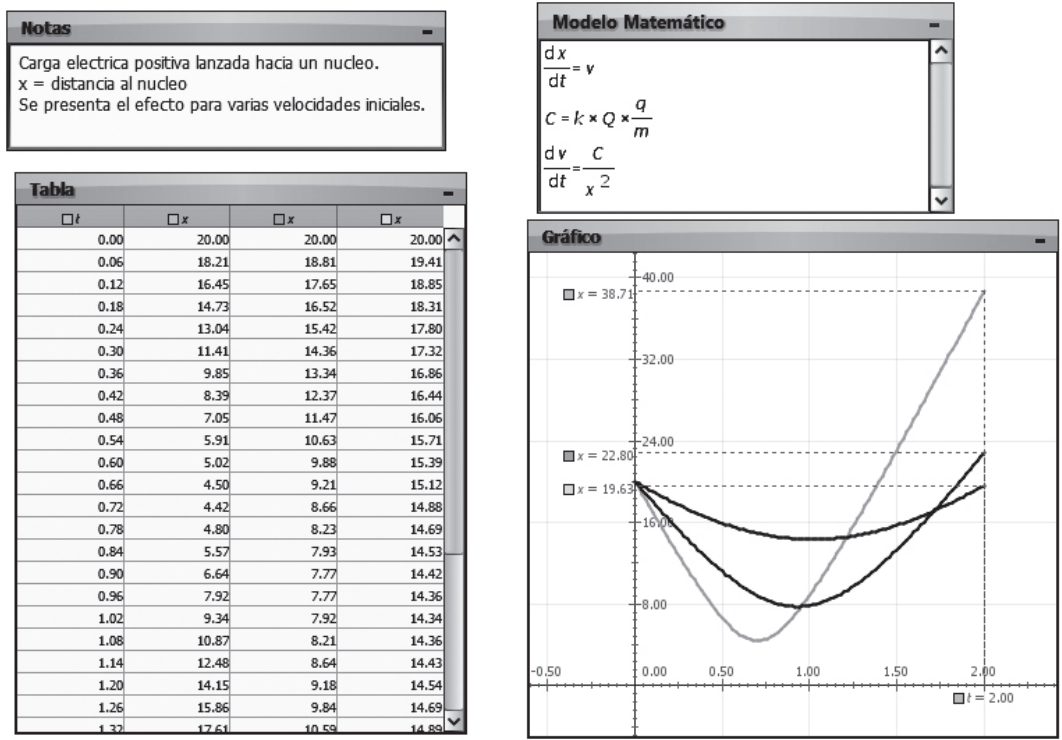

Datos utilizados: $\mathrm{q}=1,6 \times 10^{-19} \mathrm{C} ; \mathrm{Q}=10 \mathrm{q}_{1}=1,6 \times 10^{-18} \mathrm{C} ; \mathrm{m}=9,10 \times 10^{-31} \mathrm{~kg}$ Distancia inicial: $\mathrm{x}_{0}=20 \mathrm{~m}$

Parámetro: Velocidad inicial Vo $=-30 \mathrm{~m} / \mathrm{s},-20 \mathrm{~m} / \mathrm{s}$ y $-10 \mathrm{~m} / \mathrm{s}$;

De la tabla de datos obtenida se aprecia que las distancias más cercanas son de 4,42 m; 7,77 m y 14,34 m respectivamente con las velocidades. Estando entonces en condiciones de realizar un análisis mucho mayor sobre el experimento.

\section{Fuerzas dependientes de la velocidad. Fricción en fluidos. $F=-k v^{n}$}

Una esfera se desplaza dentro de un fluido viscoso. (Sears - Zemansky, 2009)

Segunda Ley de Newton: $-k v=m \frac{d^{2} x}{d t^{2}}$

Ecuación diferencial por resolver: $\frac{d^{2} x}{d t^{2}}+c v=0 ;$ siendo $c=\frac{2 \pi R \eta}{m}$ 


\begin{tabular}{|c|c|c|c|c|c|}
\hline \multicolumn{6}{|c|}{ Notas } \\
\hline \multicolumn{6}{|c|}{$\begin{array}{l}\text { Movimiento de un cuerpo dentro de } \\
\text { viscoso. } \\
\text { Se aprecia el fecto de la viscosidad de } \\
\text { movimiento de la esfera. }\end{array}$} \\
\hline \multicolumn{6}{|c|}{ Tabla } \\
\hline$\square t$ & $\square v$ & $\square v$ & $\square v$ & $\square v$ & \\
\hline 0.00 & 10.00 & 10.00 & 10.00 & 10.00 & $\wedge$ \\
\hline 0.10 & 8.82 & 8.28 & 7.78 & 7.30 & \\
\hline 0.20 & 7.78 & 6.86 & 6.05 & 5.34 & \\
\hline 0.30 & 6.86 & 5.68 & 4.71 & 3.90 & \\
\hline 0.40 & 6.05 & 4.70 & 3.66 & 2.85 & \\
\hline 0.50 & 5.33 & 3.90 & 2.85 & 2.08 & \\
\hline 0.60 & 4.70 & 3.23 & 2.21 & 1.52 & \\
\hline 0.70 & 4.15 & 2.67 & 1.72 & 1.11 & \\
\hline 0.80 & 3.66 & 2.21 & 1.34 & 0.81 & \\
\hline 0.90 & 3.23 & 1.83 & 1.04 & 0.59 & \\
\hline 1.00 & 2.85 & 1.52 & 0.81 & 0.43 & \\
\hline 1.10 & 2.51 & 1.26 & 0.63 & 0.32 & \\
\hline 1.20 & 2.21 & 1.04 & 0.49 & 0.23 & \\
\hline 1.30 & 1.95 & 0.86 & 0.38 & 0.17 & $\checkmark$ \\
\hline
\end{tabular}
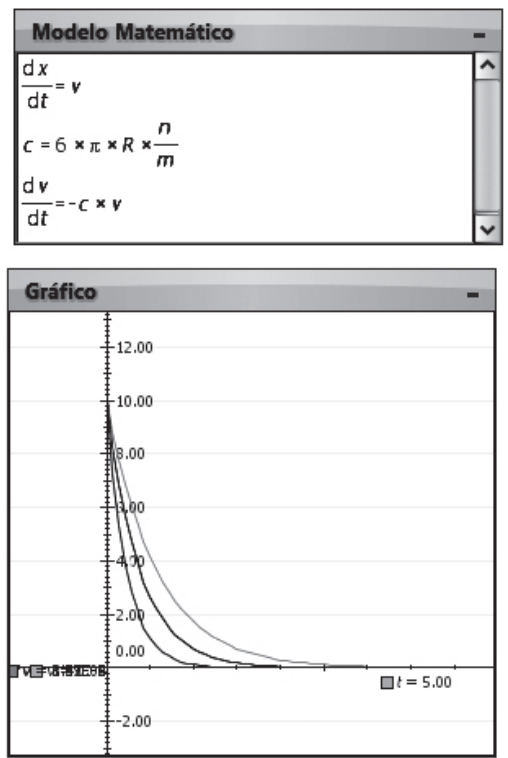

Datos utilizados:

Radio de la esfera: $\mathrm{R}=0,020 \mathrm{~m}$

Masa de la esfera: $\mathrm{m}=0,30 \mathrm{~kg}$

Velocidad inicial: $\mathrm{v}_{0}=10,0 \mathrm{~m} / \mathrm{s}$

Parámetro: Viscosidad del fluido: $\eta=1,0$ Pa.s; 2,0 Pa.s y 2,5 Pa.s

De la tabla de datos obtenida se aprecia la reducción de la velocidad para diferentes valores de la viscosidad del fluido. Estando entonces en condiciones de realizar un análisis mucho mayor sobre el experimento.

\section{Movimiento oscilatorio simple}

Un bloque de masa $\mathrm{M}$, atado a un resorte realiza un movimiento oscilatorio. (Sears - Zemansky, 2009)

Segunda ley de Newton: $-k x=m \frac{d^{2} x}{d t^{2}}$

Ecuación diferencial: $\frac{d^{2} x}{d t^{2}}+c x=0$; siendo $c=\frac{k}{m}$ 


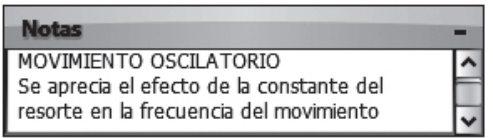

\begin{tabular}{|c|c|c|c|}
\hline \multicolumn{4}{|l|}{ Tabla } \\
\hline ㅁt & $\square x$ & $\square x$ & $\square x$ \\
\hline 0.00 & 5.00 & 5.00 & $5 . 0 0 \longdiv { \wedge }$ \\
\hline 0.10 & 4.90 & 4.85 & 4.80 \\
\hline 0.20 & 4.61 & 4.41 & 4.22 \\
\hline 0.30 & 4.13 & 3.71 & 3.31 \\
\hline 0.40 & 3.48 & 2.79 & 2.13 \\
\hline 0.50 & 2.70 & 1.70 & 0.78 \\
\hline 0.60 & 1.81 & 0.50 & -0.63 \\
\hline 0.70 & 0.85 & -0.72 & -1.99 \\
\hline 0.80 & -0.15 & -1.90 & -3.19 \\
\hline 0.90 & -1.14 & -2.96 & -4.14 \\
\hline 1.00 & -2.08 & -3.85 & -4.76 \\
\hline 1.10 & -2.94 & -4.51 & -5.00 \\
\hline 1.20 & -3.69 & -4.90 & -4.84 \\
\hline 1.30 & -4.28 & -5.00 & $-4,30 \sqrt{\checkmark}$ \\
\hline
\end{tabular}
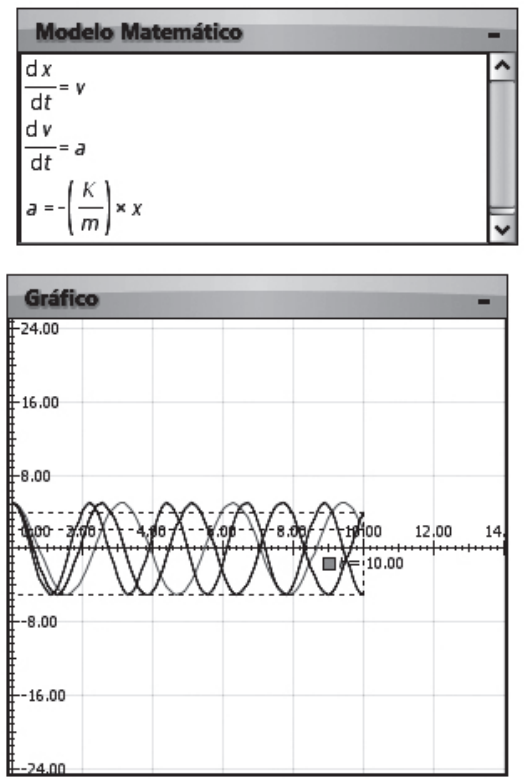

Datos utilizados:

Masa del bloque: $\mathrm{M}=2,0 \mathrm{~kg}$

Posición inicial: $\mathrm{x}_{0}=5,0 \mathrm{~m}$

Velocidad inicial: $\mathrm{v}_{0}=0$ (Parte del reposo.)

Parámetro Constante elástica del resorte: $\mathrm{k}=20$ N/m, 30 N/m y 40 N/m

De la tabla de datos obtenida se aprecia la obtención de un m.a.s. y el efecto de los valores de la constante del resorte en la frecuencia de oscilación. Estando entonces en condiciones de realizar un análisis mucho mayor sobre el experimento.

\section{Movimiento oscilatorio amortiguado}

Un bloque de masa $\mathrm{M}$, atado a un resorte realiza un movimiento oscilatorio bajo la presencia de una fuerza de fricción producida por un fluido viscoso. (Sears - Zemansky, 2009)

Segunda Ley de Newton: $-k x-b \frac{d x}{d t}=m \frac{d^{2} x}{d t^{2}}$ 
Ecuación diferencial: $\frac{d^{2} x}{d t^{2}}+r \frac{d x}{d t}+c x=0$; siendo, $c=\frac{k}{m} r=\frac{b}{m}$
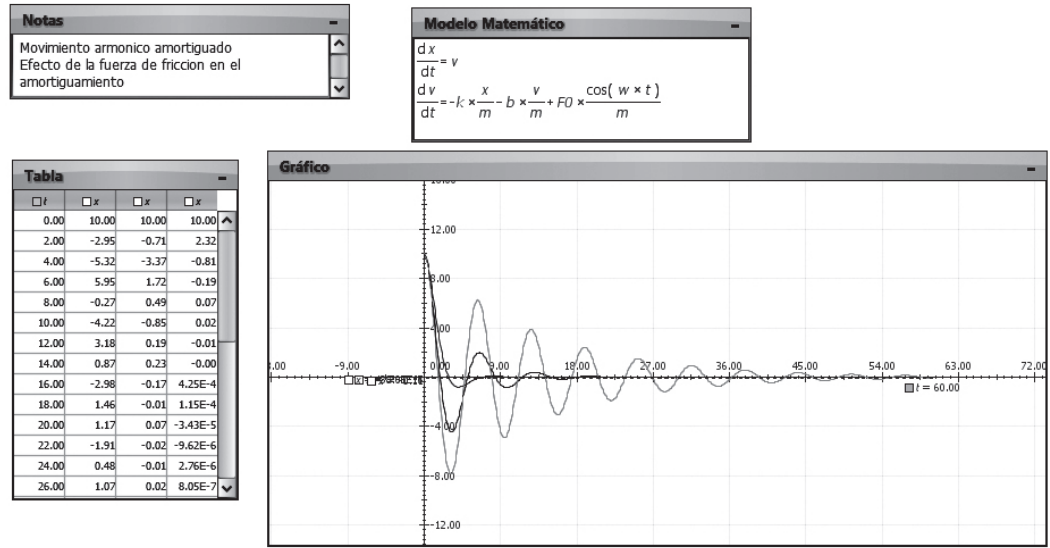

Datos utilizados:

Masa del bloque: $\mathrm{M}=20 \mathrm{~kg}$

Constante elástica del resorte: $\mathrm{K}=20 \mathrm{~N} / \mathrm{m}$

Posición inicial: $\mathrm{x}_{0}=10 \mathrm{~m}$

Velocidad inicial: $\mathrm{v}_{0}=0$ (Parte del reposo.)

Parámetro Coeficiente de amortiguación: $\mathrm{b}=3,0 \mathrm{~kg} / \mathrm{s}, 10 \mathrm{~kg} / \mathrm{s}$ y $25 \mathrm{~kg} / \mathrm{s}$

De la tabla de datos obtenida se aprecia la amortiguación leve, fuerte y extrema en el movimiento dependiendo de los valores del coeficiente de amortiguación respectivamente. Estando entonces en condiciones de realizar un análisis mucho mayor sobre el experimento.

\section{Movimiento oscilatorio amortiguado forzado}

Un bloque de masa $\mathrm{M}$, atado a un resorte realiza un movimiento oscilatorio bajo la presencia de una fuerza de fricción producida por un fluido viscoso y una fuerza externa periódica. (Sears - Zemansky, 2009)

Segunda ley de Newton: $-k x-b v+$ Focoswt $=m \frac{d^{2} x}{d t^{2}}$ 
Ecuación diferencial: $\frac{d^{2} x}{d t^{2}}=-\left(\frac{k}{m}\right) x-\left(\frac{b}{m}\right) \frac{d x}{d t}+\left(\frac{F_{0}}{m}\right) \cos w t$

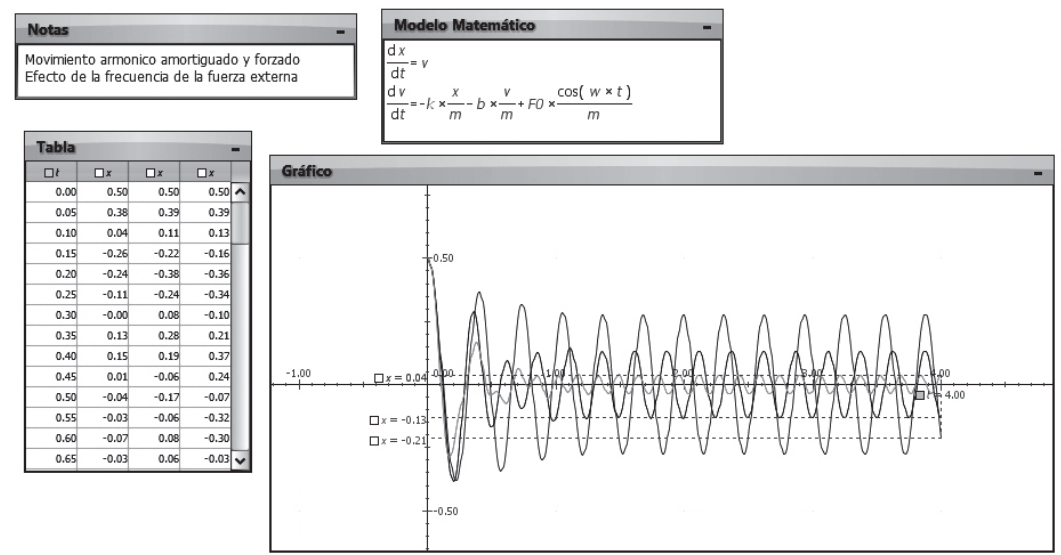

Datos utilizados:

Masa del bloque: $\mathrm{m}=2,0 \mathrm{~kg}$

Constante elástica del resorte: $\mathrm{k}=600 \mathrm{~N} / \mathrm{m}$

Coeficiente de amortiguación: $\mathrm{b}=15 \mathrm{~kg} / \mathrm{s}$

Posición inicial: $\mathrm{x}_{0}=0,50 \mathrm{~m}$

Velocidad inicial: $\mathrm{v}_{0}=0$ (Parte del reposo.)

Amplitud de fuerza externa: $\mathrm{F}_{0}=100 \mathrm{~N}$

Parámetro: Frecuencia de la fuerza externa $\mathrm{w}=40,0 \mathrm{rad} / \mathrm{s}, 25,0 \mathrm{rad} / \mathrm{s}$ y $20,0 \mathrm{rad} / \mathrm{s}$

De la tabla de datos obtenida se aprecia inicialmente un movimiento transitorio que se desvanece en el tiempo quedando un movimiento oscilatorio y que la amplitud del movimiento oscilatorio final depende de la frecuencia de la fuerza externa y de otros parámetros. Estando entonces en condiciones de realizar un análisis mucho mayor sobre el experimento.

\section{Movimiento pendular}

Una masa m suspendida de una cuerda liviana de longitud L, realizan un movimiento periódico para cualquier ángulo de inclinación inicial. (Sears - Zemansky, 2009) 
Segunda ley de Newton: $-m g \operatorname{sen} \theta=m \frac{d^{2}(L \theta)}{d t^{2}}$

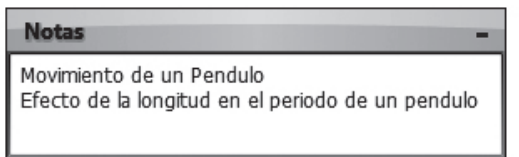

Efecto de la longitud en el periodo de un pendulo

\begin{tabular}{|c|c|c|c|c|}
\hline \multicolumn{4}{|l|}{ Tabla } & \multirow[t]{2}{*}{ - } \\
\hline$\square t$ & $\square x$ & $\square x$ & $\square x$ & \\
\hline 0.00 & 1.50 & 1.50 & 1.50 & 1 \\
\hline 0.10 & 1.47 & 1.48 & 1.49 & \\
\hline 0.20 & 1.37 & 1.43 & 1.46 & \\
\hline 0.30 & 1.21 & 1.35 & 1.40 & \\
\hline 0.40 & 0.99 & 1.24 & 1.33 & \\
\hline 0.50 & 0.71 & 1.10 & 1.23 & \\
\hline 0.60 & 0.39 & 0.92 & 1.11 & \\
\hline 0.70 & 0.05 & 0.72 & 0.98 & \\
\hline 0.80 & -0.30 & 0.50 & 0.82 & \\
\hline 0.90 & -0.63 & 0.27 & 0.65 & \\
\hline 1.00 & -0.92 & 0.02 & 0.47 & \\
\hline 1.10 & -1.15 & -0.22 & 0.27 & \\
\hline 1.20 & -1.33 & -0.46 & 0.07 & \\
\hline 1.30 & -1.45 & -0.68 & -0.13 & $v$ \\
\hline
\end{tabular}
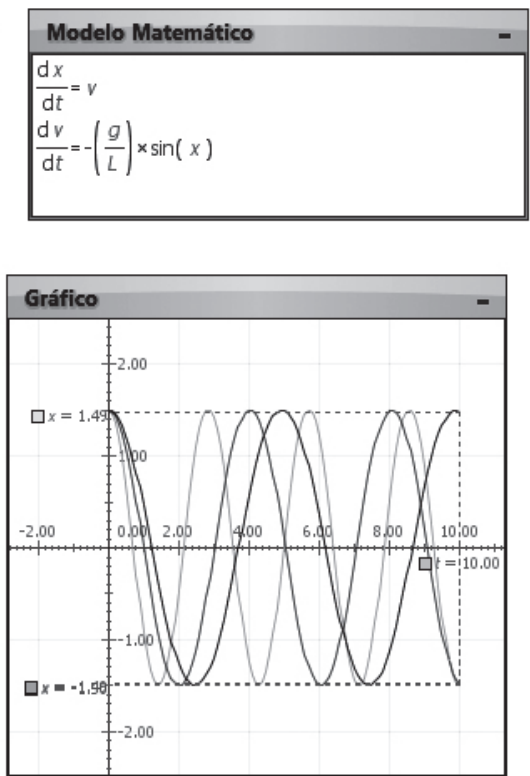

Ecuación diferencial: $\frac{d^{2} \theta}{d t^{2}}+g \operatorname{sen} \theta=0$

Datos utilizados:

Aceleración de la gravedad: $\mathrm{g}=9,81 \mathrm{~m} / \mathrm{s}^{2}$

Posición inicial: $\theta_{0}=1,5 \mathrm{rad}$

Velocidad inicial: $\mathrm{v}_{0}=0$ (Parte del reposo.)

Parámetro: Longitud de la cuerda, $\mathrm{L}=1,5 \mathrm{~m} ; 3,0 \mathrm{~m}$ y 4,5 m

De la tabla de datos obtenida se aprecia el cambio en la frecuencia y del periodo de oscilación en función de la longitud del péndulo. Estando entonces en condiciones de realizar un análisis mucho mayor sobre el experimento. 
7. Movimiento de una carga eléctrica bajo la acción de un campo eléctrico uniforme, perpendicular a su velocidad inicial.

Segunda ley de Newton: $q \vec{E}=m \frac{d^{2} \bar{r}}{d t^{2}}$

Ecuaciones diferenciales: Eje X: $0=\mathrm{m} \frac{d^{2} x}{d t^{2}} ; \frac{d^{2} x}{d t^{2}}=0$

\begin{tabular}{|c|c|c|c|}
\hline \multicolumn{4}{|l|}{ Notas } \\
\hline \multicolumn{4}{|c|}{$\begin{array}{l}\text { Movimiento de carga electrico dentro } \\
\text { campo electrico vertical. } \\
\text { Efecto del campo electrico en el mov } \\
\text { carga }\end{array}$} \\
\hline \multicolumn{4}{|l|}{ Tabla } \\
\hline ㅁt & ㅁy & $\square y$ & 口y \\
\hline 0.00 & 0.00 & 0.00 & $0 . 0 0 \longdiv { \wedge }$ \\
\hline 0.10 & 1.50 & 1.49 & 1.49 \\
\hline 0.20 & 2.98 & 2.96 & 2.95 \\
\hline 0.30 & 4.46 & 4.42 & 4.38 \\
\hline 0.40 & 5.93 & 5.86 & 5.79 \\
\hline 0.50 & 7.39 & 7.28 & 7.17 \\
\hline 0.60 & 8.84 & 8.68 & 8.53 \\
\hline 0.70 & 10.28 & 10.07 & 9.85 \\
\hline 0.80 & 11.72 & 11.44 & 11.16 \\
\hline 0.90 & 13.14 & 12.79 & 12.43 \\
\hline 1.00 & 14.56 & 14.12 & 13.68 \\
\hline 1.10 & 15.97 & 15.44 & 14.90 \\
\hline 1.20 & 17.37 & 16.73 & 16.10 \\
\hline 1.30 & 18.76 & 18.01 & 17.27 V \\
\hline
\end{tabular}
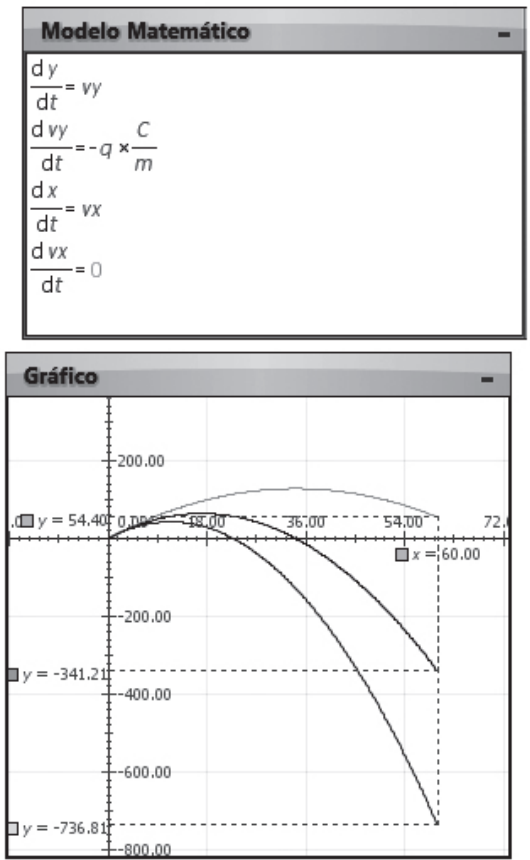

Eje Y: $-\mathrm{qE}=\mathrm{m} \frac{d^{2} y}{d t^{2}} ; \frac{d^{2} y}{d t^{2}}=-c$; siendo $c=\frac{q E}{m}$

Datos utilizados:

Carga eléctrica: $\mathrm{q}=1,6 \times 10^{-19} \mathrm{C}$

Masa de la partícula: $\mathrm{m}=9,1 \times 10^{-31} \mathrm{~kg}$

Posición inicial: $\mathrm{x}_{0}=0, \mathrm{y}_{0}=0$

Velocidad inicial: $\mathrm{vx}=2,0 \mathrm{~m} / \mathrm{s} ; \mathrm{vy}=0$

Parámetro: Campo Eléctrico: $\mathrm{E}=5,0 \times 10^{-12} \mathrm{~V} / \mathrm{m} ; 10,0 \times 10^{-12} \mathrm{~V} / \mathrm{m}$ y $15 \times 10^{-}$

$12 \mathrm{~V} / \mathrm{m}$ 
De la tabla de datos obtenida se aprecia el cambio en el recorrido de la partícula por la acción de campo eléctrico en sus diferentes valores. Estando entonces en condiciones de realizar un análisis mucho mayor sobre el experimento.

\section{Carga y descarga de un condensador}

Cuando un condensador eléctrico inicialmente descargado, se conecta a una batería a través de una resistencia eléctrica, se inicia el proceso de acumulación de carga eléctrica en el condensador llegando asintóticamente hasta una carga final, dependiendo de la capacitancia del condensador. (Sears - Zemansky - Young - Freedman. 2005)

a) Para el proceso de carga se tienen las siguientes ecuaciones:

Segunda ley de Kirchhoff: $\in-R \frac{d q}{d t}-\frac{q}{c}=0$

Ecuación diferencial: $\frac{q}{c}+R \frac{d q}{d t}=\epsilon$

b) Para el proceso de descarga, en las ecuaciones anteriores hacemos $\epsilon=0$.

Segunda ley de Kirchhoff: $-R \frac{d q}{d t}-\frac{q}{c}=0$

Ecuación diferencial: $\frac{q}{c}+R \frac{d q}{d t}=0$

Datos utilizados:

Resistencia eléctrica: $\mathrm{R}=20 \Omega$

Parámetro: Capacidad del condensador: $\mathrm{C}=0,50 \mathrm{~F} ; 0,30 \mathrm{~F}$ y $0,10 \mathrm{~F}$

Fuerza electromotriz de la batería: $\epsilon=20 \mathrm{~V}$

Carga eléctrica inicial del condensador: $\mathrm{q}=0$ 


\begin{tabular}{|l|l|}
\hline Notas \\
\hline Poceso de carga de un condensador. \\
Efecto de la capacitancia en en el proceso de \\
carga del condensador.
\end{tabular}

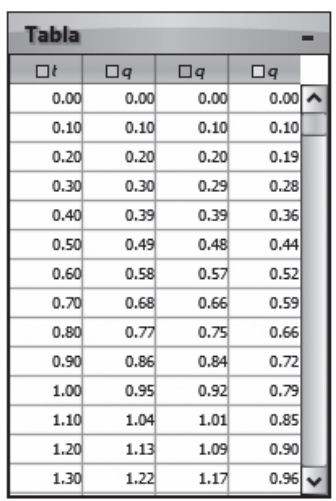
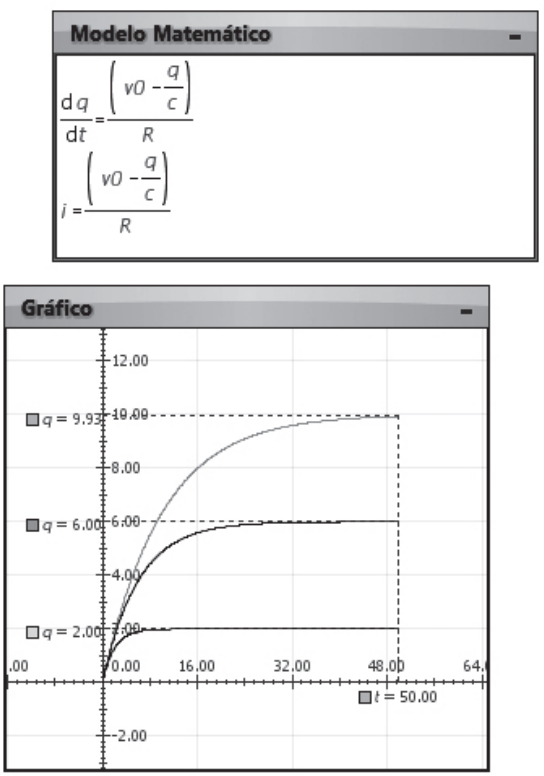

Carga eléctrica inicial del condensador: $\mathrm{q}=10 \mathrm{C}$
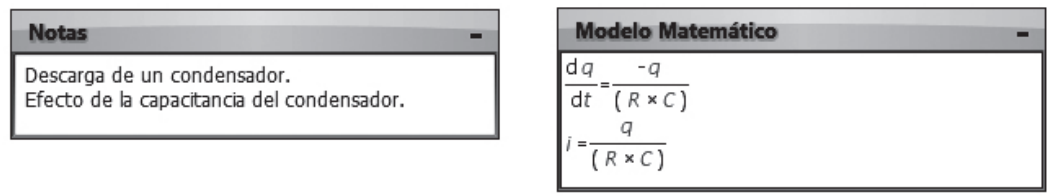

\begin{tabular}{|c|c|c|c|c|}
\hline \multicolumn{4}{|l|}{ Tabla } & $=$ \\
\hline$\square t$ & $\square q$ & $\square q$ & $\square q$ & \\
\hline 0.00 & 10.00 & 10.00 & 10.00 & 1 \\
\hline 0.10 & 9.90 & 9.83 & 9.51 & \\
\hline 0.20 & 9.80 & 9.67 & 9.05 & \\
\hline 0.30 & 9.70 & 9.51 & 8.61 & \\
\hline 0.40 & 9.61 & 9.36 & 8.19 & \\
\hline 0.50 & 9.51 & 9.20 & 7.79 & \\
\hline 0.60 & 9.42 & 9.05 & 7.41 & \\
\hline 0.70 & 9.32 & 8.90 & 7.05 & \\
\hline 0.80 & 9.23 & 8.75 & 6.70 & \\
\hline 0.90 & 9.14 & 8.61 & 6.38 & \\
\hline 1.00 & 9.05 & 8.46 & 6.07 & \\
\hline 1.10 & 8.96 & 8.32 & 5.77 & \\
\hline 1.20 & 8.87 & 8.19 & 5.49 & \\
\hline 1.30 & 8.78 & 8.05 & 5.22 & 2 \\
\hline
\end{tabular}

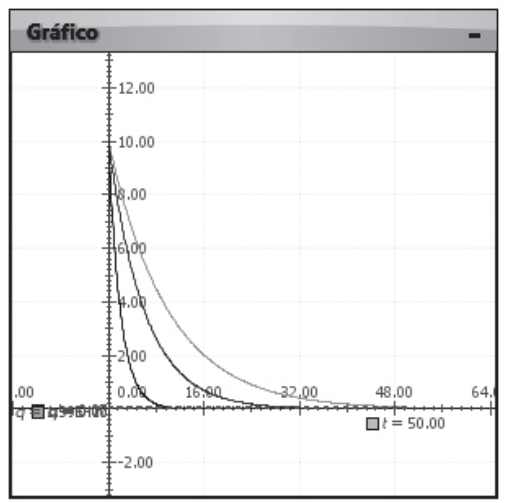


De la tabla de datos obtenida se aprecia el efecto de amortiguación que ofrece la capacitancia del condensador para ambos procesos de carga y descarga. Estando entonces en condiciones de realizar un análisis mucho mayor sobre el experimento.

\section{Circuitos RLC}

En un circuito RLC en serie con un generador eléctrico, se presenta primero un efecto transitorio y luego una oscilación permanente tanto para la corriente, carga en el condensador y los voltajes en el circuito eléctrico. (Sears - Zemansky - Young - Freedman. 2005)

Segunda ley de Kirchhoff: $\varepsilon s e n w t-i R-L \frac{d i}{d t}-\frac{q}{C}=0$

Ecuación diferencial: $\frac{d^{2} v}{d t^{2}}+\frac{R}{L} \frac{d v}{d t}+\frac{v}{L C}=\frac{\varepsilon}{L C}$ senwt

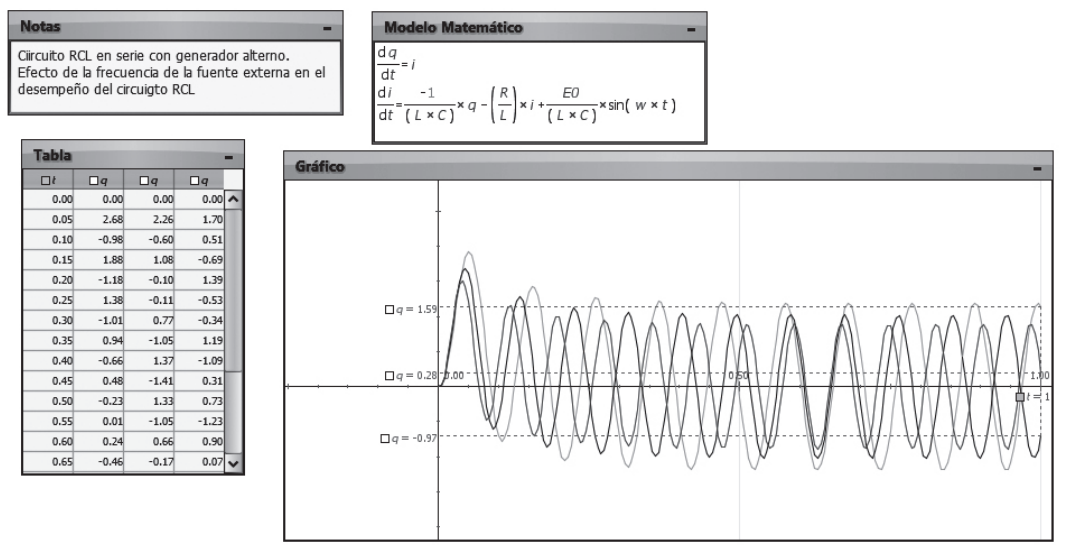

Datos utilizados:

Inductancia de la bobina: $\mathrm{L}=0,03 \mathrm{H}$

Capacitancia del condensador: $\mathrm{C}=0,01 \mathrm{~F}$

Resistencia eléctrica: $\mathrm{R}=10 \Omega$

Fem pico del generador: $\epsilon_{0}=10 \mathrm{~V}$

Parámetro: Frecuencia del generador $\mathrm{w}=60 \mathrm{~Hz}, 70 \mathrm{~Hz}$ y $80 \mathrm{~Hz}$ 
De la tabla de datos obtenida se aprecia primero un estado transitorio y luego un estado oscilante estable. La frecuencia del oscilador influye en la amplitud de las cantidades eléctricas (carga, corriente y voltaje). Estando entonces en condiciones de realizar un análisis mucho mayor sobre el experimento.

\section{RESULTADOS}

Podemos apreciar que sin utilizar procesos de cálculo tediosos y sin utilizar soluciones analíticas, es posible estudiar y comprender fenómenos físicos, recurriendo solo a unas cuantas sentencias de programación requerida por el software.

\section{CONCLUSIONES}

De la misma forma como actualmente prescindimos de cálculos que se hacían manualmente, en la medida que la tecnología nos ha facilitado los resultados como por ejemplo la obtención de raíz cuadrada, cubica, solución de ecuaciones algebraicas, etc.; también podemos ir avanzando y utilizar los softwares, muchos de ellos gratuitos como el usado en el presente trabajo, y prescindir de los tediosos cálculos de integración y solución de ecuaciones diferenciales, en la enseñanza y aprendizaje de la física.

Esta forma de apreciar la metodología de resolver modelos físicos que representan a fenómenos presentados en la naturaleza, ha permitido a la ciencia y tecnología resolver casos cada vez mucho más complejos $\mathrm{y}$ ahora es nuestro compromiso incorporarlo formalmente en el proceso de enseñanza - aprendizaje de la física tanto a nivel universitario como escolar. 


\section{LITERATURA CITADA}

VITOR DUARTE TEODORO, JOAO PAULO DUQUE VIEIRA, FILIPE COSTA CLERIGO. 1996. Introducción a Modellus.

SEARS - ZEMANSKY. 2009. Física Universitaria. Volumen 1.

SEARS - ZEMANSKY - YOUNG - FREEDMAN. 2005. Física Universitaria. Volumen 2. 